\title{
Relationship between bite size per mouthful and dental arch size in healthy subjects
}

\author{
Kouichi Shiozawa ${ }^{1}$ - Yasumasa Mototani ${ }^{1} \cdot$ Kenji Suita $^{1}$ - Aiko Ito $^{1,2} \cdot$ Naoya Kawamura $^{1,3} \cdot$ Yuka Yagisawa $^{1,2}$. \\ Ichiro Matsuo ${ }^{1,3} \cdot$ Yoshio Hayakawa $^{1,4} \cdot$ Megumi Nariyama $^{5}$. Daisuke Umeki ${ }^{2}$. Yasutake Saeki ${ }^{1}$. Yoshiki Ohnuki ${ }^{1}$. \\ Satoshi Okumura ${ }^{1}$ (1)
}

Received: 12 April 2018 / Accepted: 17 July 2018 / Published online: 28 July 2018

(c) The Physiological Society of Japan and Springer Japan KK, part of Springer Nature 2018

\begin{abstract}
Although multiple factors influence food bite size, the relationship between food bite size per mouthful and mandible or tongue size remains poorly understood. Here, we examined the correlations between food bite size and the lower dental arch size (an indicator of tongue size) in human subjects with good oral and general health, using fish sausage and bread as test foods. Notably, bite size of both foods was significantly positively correlated with the lower dental arch size, whereas masticatory performance (measured in terms of glucose extraction from a gummy jelly) showed no dependence on bite size. Further, bite size was significantly positively correlated with the body mass index. Our findings suggest that larger bite size is associated with larger tongue size, which might be a contributory factor to obesity.
\end{abstract}

Keywords Bite size $\cdot$ Dental arch size $\cdot$ Tongue size $\cdot$ Masticatory performance

\section{Introduction}

Major oral physiological factors that regulate bite size and chewing include the cycle of jaw and tongue movements [1, 2]. An autopsy study demonstrated that the human tongue has a high percentage of fat, and indicated that tongue weight and tongue fat percentage are closely associated with the degree of obesity [3]. Videofluorography studies in

Electronic supplementary material The online version of this article (https://doi.org/10.1007/s12576-018-0630-8) contains supplementary material, which is available to authorized users.

Satoshi Okumura

okumura-s@tsurumi-u.ac.jp

1 Department of Physiology, Tsurumi University School of Dental Medicine, 2-1-3 Tsurumi, Tsurumi-ku, Yokohama 230-8501, Japan

2 Department of Orthodontics, Tsurumi University School of Dental Medicine, Yokohama 230-8501, Japan

3 Department of Periodontology, Tsurumi University School of Dental Medicine, Yokohama 230-8501, Japan

4 Department of Dental Anesthesiology, Tsurumi University School of Dental Medicine, Yokohama 230-8501, Japan

5 Department of Pediatric Dentistry, Tsurumi University School of Dental Medicine, Yokohama 230-8501, Japan human subjects indicated that a preparatory forward movement of the tongue to receive food occurs during food intake, followed by retraction of the tongue to introduce the food into the mouth, and it was reported that tongue manipulation plays an important role in recognizing and evaluating the volume of the bite taken [4].

Based on the above reports, we hypothesized that bite size per mouthful might be positively correlated with tongue size. In this work, we tested this idea in healthy human subjects. We employed the size of the lower dental arch as a surrogate for tongue size because previous studies have established the morphologic relationship between the tongue size and the lower dental arch size [5, 6]. More importantly, direct measurements of tongue size by computerized tomography or magnetic resonance imaging are subject to artifacts due to metal prostheses, and it is also ethically questionable to expose subjects to medically unnecessary imaging procedures. In addition, we examined the correlation between bite size and body mass index (BMI), a useful indicator of obesity, because the relationship between the two still remains controversial [7-11]. 


\section{Materials and methods}

\section{Subjects}

Sixty-one adult subjects (39 males, 22 females, mean age 23.3) were randomly selected from the students, postgraduate students, and staff in Tsurumi University School of Dental Medicine according to the following criteria: good oral and general health; no pain during chewing; complete natural dentition without any stomatognathic problems; not taking medication that might affect mastication or insalivation; no history of orthodontic treatments. The height and weight of subjects were measured, and BMI was calculated by dividing the body weight $(\mathrm{kg})$ by the height squared $\left(\mathrm{m}^{2}\right)$.

\section{Experimental procedure}

Fish sausage $(\mathrm{FS})($ diameter $=20 \mathrm{~mm}$, length $=100 \mathrm{~mm}$; Osakana-sausage, Nissui, Tokyo, Japan) (Fig. 1a-1) and bread (B) (length $=100 \mathrm{~mm}$, width $=40 \mathrm{~mm}$, thickness $=20$ mm; Chojyuku, Pasco, Tokyo, Japan) (Fig. 1a-2) were used in this study as test foods. Subjects were asked to take one bite of each sample with a single occlusion and to chew as usual before swallowing (Fig. 1b). This task was repeated more than three times for each test food, and the foods were served in a random order [8]. Bite size per mouthful was calculated from the food weights (gram: g) before and after each bite, measured on a microscale.

\section{Masticatory performance}

Subjects were asked to chew a cylindrically shaped 5\% glucose-containing gummy jelly with a diameter of $14 \mathrm{~mm}$, height of $10 \mathrm{~mm}$, and weight of $2.3 \mathrm{~g}$ (Glucorumn, GC Corporation, Tokyo, Japan) (Fig. 1c-1) for $20 \mathrm{~s}$ on their habitual chewing side $[12,13]$. After chewing, the subjects were asked to hold $10 \mathrm{ml}$ of distilled water (Fig. 1c-3) in their mouth briefly and then to split into a cup equipped with a filter (Fig. 1c-2). The glucose concentration in the filtrate was measured using a glucose measuring device (Fig. 1c-4) (Glucosensor GS-1, GC, Tokyo, Japan), and the amount of glucose extraction was used as a quantitative parameter of the masticatory performance [12-14].

\section{Measurement of the lower dental arch size}

Paraffin wax bite impressions (Base Plate Paraffin Wax, GC Corporation, Tokyo, Japan) were obtained in each subject and the following parameters were measured in the present study (Fig. 1d).

1. Lower dental arch width: distance between the lowest margin of the lingual surface of the right and left first premolars.

2. Lower dental arch length: distance from the incisal edge of the central incision to the line that passes through the distal edge of the first molars.
Fig. 1 a, b Fish sausage (left) and bread (right) were used in this study as test foods (a) and the experiments were performed as shown in (b). c Masticatory performance of objects was examined by requesting them to chew a cylindrically shaped $5 \%$ glucose-containing gummy jelly (c-1) for $20 \mathrm{~s}$. After chewing, the subjects were asked to hold $10 \mathrm{ml}$ of distilled water (c-3) in their mouth briefly and then split into a cup equipped with a filter (c-2). The glucose concentration in the filtrate was measured using a glucose measuring device (c-4), and the amount of glucose extraction was used as a quantitative measure of masticatory performance. d Paraffin wax bite impression of each subject was obtained to measure the lower dental arch width and length
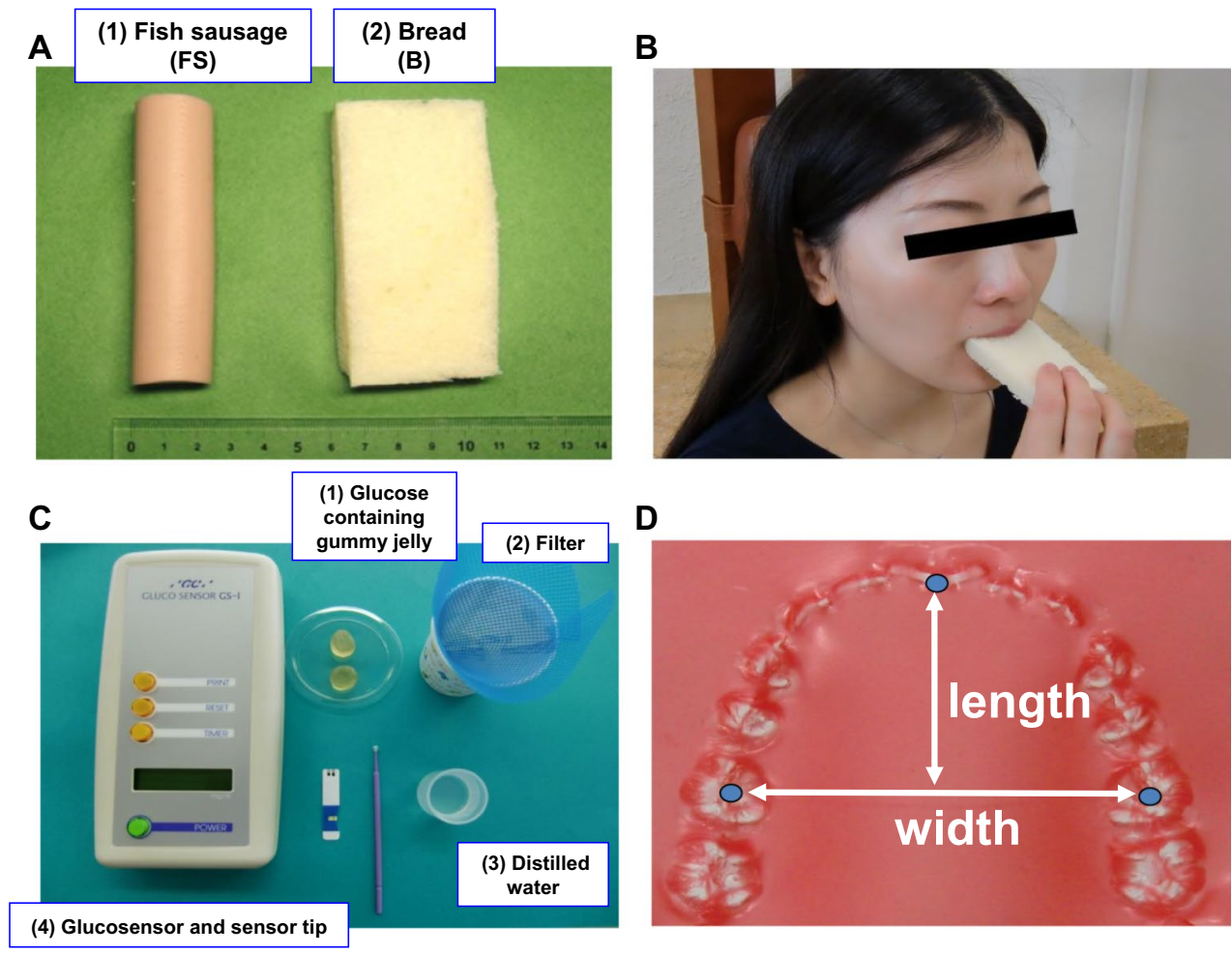


\section{Statistical analysis}

The correlations and regression lines between bite size and each parameter [lower dental arch size (width and length) (Fig. 2), glucose extraction (Fig. 3) and BMI (Supplemental Figure 1)] were determined by calculating Spearman's rank correlation coefficient in SPSS software version 13.0 (SPSS Inc., Chicago, IL, USA). Correlation was considered statistically significant when $P<0.05$.
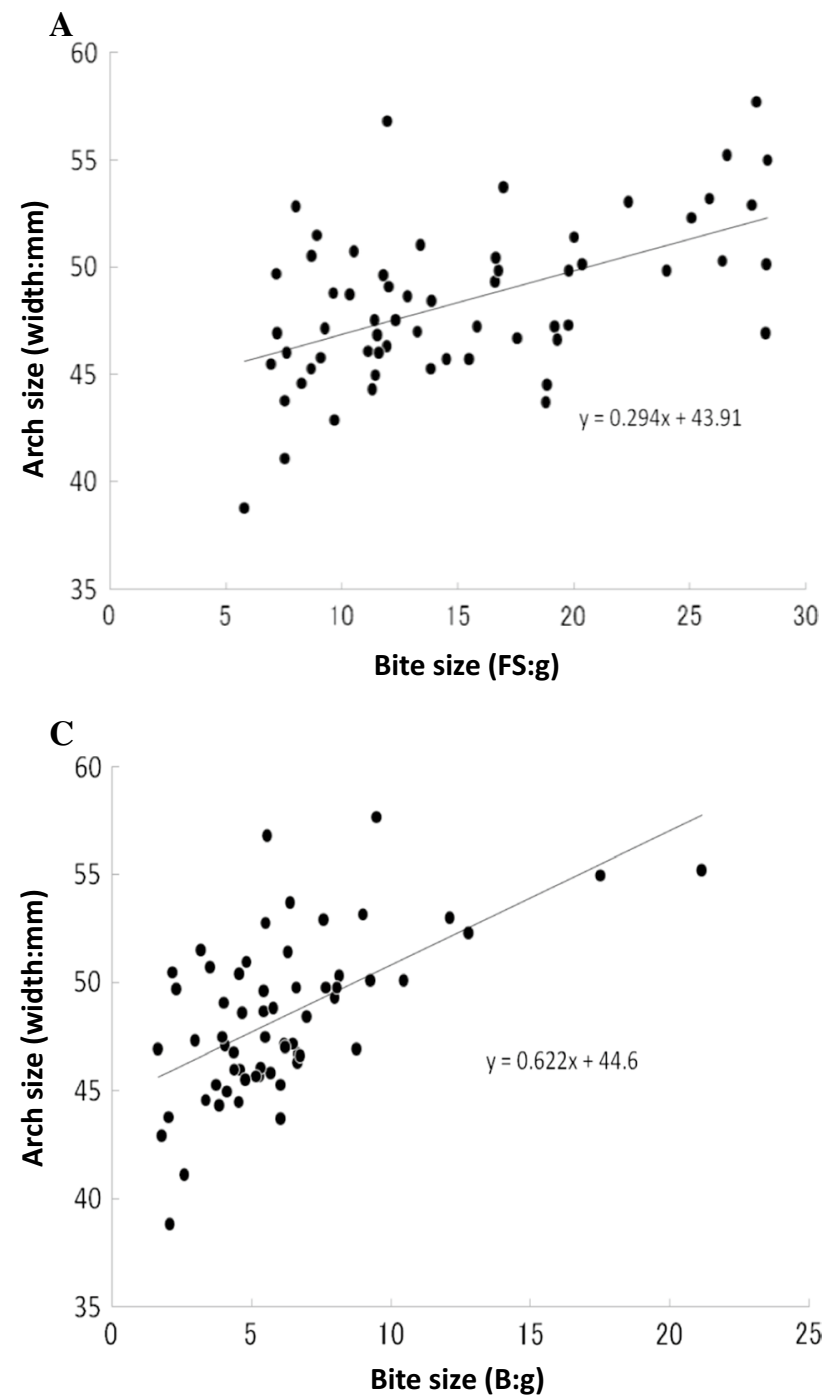

Fig. 2 Relationship between bite size and lower dental arch size. Significant correlations were observed between the bite size and the lower dental arch width $(P<0.001, r=0.490)$ (a) and length $(P<0.001, r=0.504)(\mathbf{b})$ for fish sausage as test food. Significant cor-

\section{Results}

\section{Relationship between bite size per mouthful and tongue volume}

As lower dental arch size is significantly correlated with tongue volume [5, 6], we measured lower dental arch size (width and length) using paraffin wax bite impressions in order to examine whether bite size is related to tongue volume, and thus to obesity.

As shown in Fig. 2, significant correlations were observed between bite size and lower dental arch width $(P<0.001$, $r=0.490$ ) (Fig. 2a) as well as lower dental arch length $(P<0.001, r=0.504)$ when fish sausage was used as a test
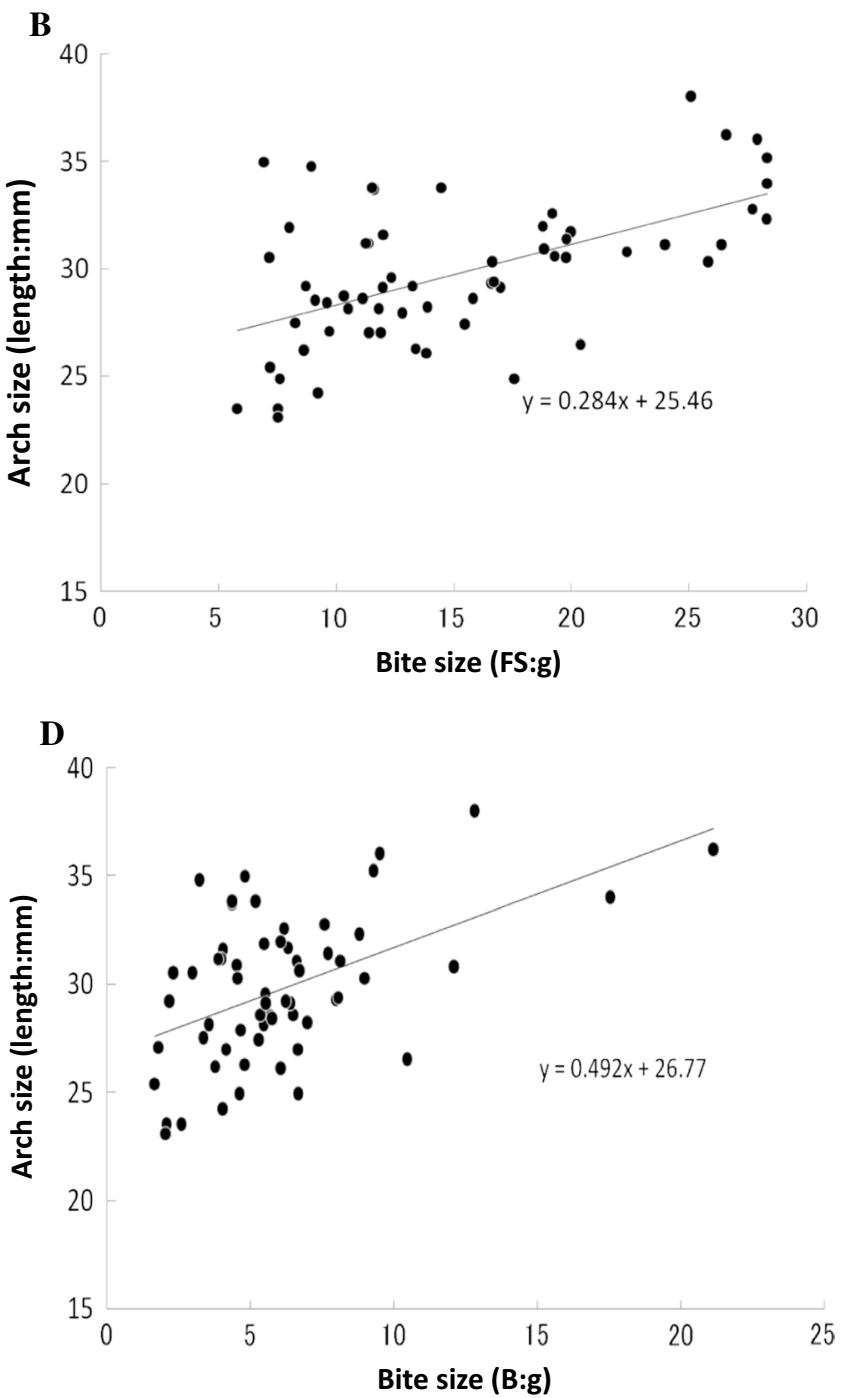

relations were also observed between bite size and lower arch dental width $(P<0.001, r=0.522)(\mathbf{c})$ and length $(P=0.001, r=0.400)(\mathbf{d})$ for bread as a test food 

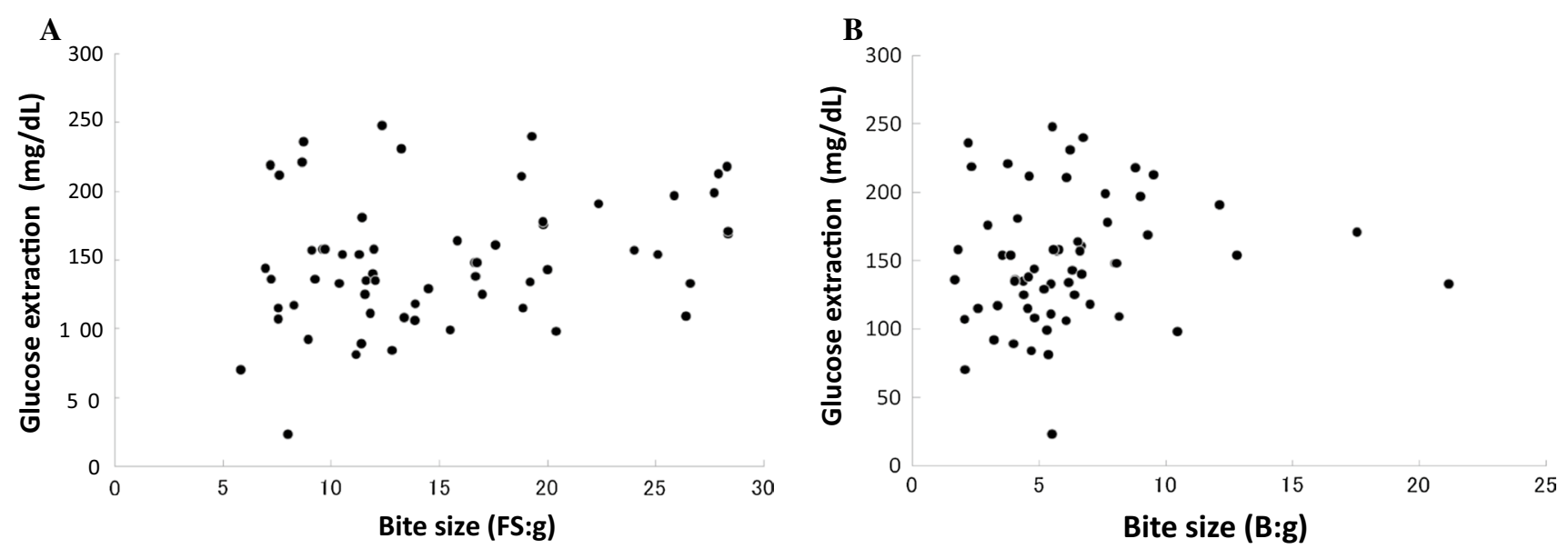

Fig. 3 Relationship between masticatory performance and the bite size for fish sausage (a) and bread (b) as test foods. No significant positive correlation was observed (fish sausage, $P=0.058, r=0.244$; bread, $P=0.077, r=0.228$ )

food (Fig. 2b). We also performed the same experiment using bread as test food and confirmed that there was a significant correlation between bite size and lower dental arch width $(P<0.001, r=0.522)$ (Fig. 2c), as well as between bite size and lower dental arch length $(P=0.001, r=0.400)$ (Fig. 2d), as in the case of fish sausage as test food.

\section{Relationship between bite size per mouthful and masticatory performance}

We next examined the influence of bite size on masticatory performance. We found no significant correlation between masticatory performance and the bite size for either fish sausage (Fig. 3a) or bread (Fig. 3b) (fish sausage, $P=0.058$, $r=0.244$; bread, $P=0.077, r=0.228$ ). Thus, masticatory performance was not affected by differences of bite size.

\section{Relationship between bite size per mouthful and body mass index}

The relationship between BMI and bite size was determined with fish sausages (Supplemental Figure 1a) and bread (Supplemental Figure 1b) as test foods. Significant positive correlations were found in both cases (fish sausage, $P=0.005$, $r=0.353$; bread, $P=0.003, r=0.369$ ).

\section{Discussion}

In the present study, we found that bite size was significantly positively correlated with the lower dental arch width or length, supporting the idea that the bite size might be positively correlated with tongue size $[5,6]$. Oral sensory exposure [15-17] and the texture of food [18-22] are thought to play important roles in regulation of food intake. We thus used two different types of food, fish sausage and bread, as test foods [23]. Similar results were obtained in both cases, suggesting that the correlations between bite size and lower dental arch size are independent of food variables.

A cross-sectional anatomic study of lingual fat in subjects from the general population at autopsy has shown that the tongue has a much higher percentage of fat than other somatic muscles, and its fat content and weight both increase in proportion to BMI [3]. The present results, together with the previous autopsy data, suggest that a larger bite size is closely associated with a larger tongue size. A possible reason for this relationship is that the tongue might play an important role in recognizing and evaluating the size of one bite of food, as suggested previously on the basis of videofluorographic analysis [4]. The present results also indicate that larger bite size might be a contributory factor to increased energy intake, which could result in obesity.

It has been shown that tongue size influences tongue neuromuscular function, tongue stiffness, and metabolic function in rats [24], but further research will be needed to explore the triadic relationship (tongue volume, bite size, and obesity) in humans in detail.

Acknowledgements We are grateful to Ms. Yoko Shinoda (Tsurumi University, Yokohama, Japan) for assistance in preparing the figures for publication.

Funding This study was supported in part by the Japan Society for the Promotion of Science (JSPS) KAKENHI Grant (15K12330 to K. Shiozawa, 22791147, 20790860 to YM, 15K18973, 24970219 to K. Suita, 17K12067 to YO, 17K11977, 26463127 to MN, 17K17342, 26861803 to DU, and 18K06862, 16H05300 to SO); MEXT-Supported Program for the Strategic Research Foundation at Private Universities (S1511018 to SO); Yokohama Academic Foundation (YO); the Naito Foundation (2015-119 to SO); Senshin Medical Research Foundation (SO); an Academic Contribution from Pfizer Japan (AC160910, AC1500818, AC170780 to S.O.); the Research Foundation for 
Community Medicine (SO); Mitsui Life Social Welfare Foundation (SO), Research Promotion Grant from the Society for Tsurumi University School of Dental Medicine (29002, 27010 to IA, 28008, 30003 to NK, 28006, 30002 to YY, 29007 to KS).

\section{Compliance with ethical standards}

Ethics approval This study was conducted with the approval of the Ethics Committee of Tsurumi University School of Dental Medicine (approval No. 1020), and followed all the principals of the Helsinki Declaration. Informed consent was obtained from each subject after a full explanation of the experimental protocol had been given.

Conflict of interest The authors report no conflict of interests.

\section{References}

1. Foster KD, Grigor JM, Cheong JN, Yoo MJ, Bronlund JE, Morgensten MP (2011) The role of oral processing in dynamic sensory perception. J Food Sci 76:R49-R61

2. Thexton AJ (1992) Mastication and swallowing: an overview. $\mathrm{Br}$ Dent J 173:197-206

3. Nashi N, Kang S, Barkdull GC, Lucas J, Davidson TM (2007) Lingual fat at autopsy. Laryngoscope 117:1467-1473

4. Okada A, Honma M, Nomura S, Yamada Y (2007) Oral behavior from food intake until terminal swallow. Physiol Behav 90:172-179

5. Tamari K, Shimizu K, Ichinose M, Nakata S, Takahama Y (1991) Relationship between tongue volume and lower dental arch sizes. Am J Orthod Dentofac Orthop 100:453-458

6. Maeda K, Tsuiki S, Isono S, Namba K, Kobayashi M, Inoue Y (2012) Difference in dental arch size between obese and nonobese patients with obstructive sleep apnoea. J Oral Rehabil 39:111-117

7. Ello-Martin JA, Ledikwe JH, Rolls BJ (2005) The influence of food portion size and energy density on energy intake: implications for weight management. Am J Clin Nutr 82:236s-241s

8. Shiozawa K, Ohnuki Y, Mototani Y, Umeki D, Ito A, Saeki Y et al (2016) Effects of food diameter on bite size per mouthful and chewing behavior. J Physiol Sci 66:93-98

9. Young LR, Nestle M (2002) The contribution of expanding portion sizes to the US obesity epidemic. Am J Public Health 92:246-249
10. Spiegel TA (2000) Rate of intake, bites, and chews-the interpretation of lean-obese differences. Neurosci Biobehav Rev 24:229-237

11. Spiegel TA, Kaplan JM, Tomassini A, Stellar E (1993) Bite size, ingestion rate, and meal size in lean and obese women. Appetite 21:131-145

12. Shiga H, Kobayashi Y, Katsuyama H, Yokoyama M, Arakawa I (2012) Gender difference in masticatory performance in dentate adults. J Prosthodont Res 56:166-169

13. Uesugi H, Shiga H (2017) Relationship between masticatory performance using a gummy jelly and masticatory movement. J Prosthodont Res 61:419-425

14. Kobayashi Y, Shiga H, Yokoyama M, Arakawa I, Nakajima K (2009) Differences in masticatory function of subjects with different closing path. J Prosthodont Res 53:142-145

15. Hetherington MM, Boyland E (2007) Short-term effects of chewing gum on snack intake and appetite. Appetite 48:397-401

16. Lavin JH, French SJ, Ruxton CH, Read NW (2002) An investigation of the role of oro-sensory stimulation in sugar satiety? Int $\mathrm{J}$ Obes Relat Metab Disord 26:384-388

17. Zijlstra N, de Wijk RA, Mars M, Stafleu A, de Graaf C (2009) Effect of bite size and oral processing time of a semisolid food on satiation. Am J Clin Nutr 90:269-275

18. de Wijk RA, Zijlstra N, Mars M, de Graaf C, Prinz JF (2008) The effects of food viscosity on bite size, bite effort and food intake. Physiol Behav 95:527-532

19. Leidy HJ, Apolzan JW, Mattes RD, Campbell WW (2010) Food form and portion size affect postprandial appetite sensations and hormonal responses in healthy, nonobese, older adults. Obesity (Silver Spring) 18:293-299

20. Mattes RD, Campbell WW (2009) Effects of food form and timing of ingestion on appetite and energy intake in lean young adults and in young adults with obesity. J Am Diet Assoc 109:430-437

21. McKiernan F, Mattes RD (2010) Effects of peanut processing on masticatory performance during variable appetitive states. J Nutr Metab 2010:487301

22. Zijlstra N, Mars M, Stafleu A, de Graaf C (2010) The effect of texture differences on satiation in 3 pairs of solid foods. Appetite 55:490-497

23. Borg P, Fogelholm M, Kukkonen-Harjula K (2004) Food selection and eating behaviour during weight maintenance intervention and 2-y follow-up in obese men. Int J Obes Relat Metab Disord 28:1548-1554

24. Brennick MJ, Delikatny J, Pack AI, Pickup S, Shinde S, Zhu JX et al (2014) Tongue fat infiltration in obese versus lean Zucker rats. Sleep 37(1095-1102):1102a-1102c 